\title{
STOPPING OF SLOW H-, He-, Li- AND Be-LIKE PROJECTILES IN ELECTRON GAS*
}

\author{
MAREK MONeta ${ }^{\dagger}$ \\ Uniwersytet Łódzki, Katedra Fizyki Ciała Stałego \\ Pomorska 149, 90-236 Łódź, Poland
}

(Received January 20, 1998; revised version March 29, 1998)

\begin{abstract}
Results of calculations of the electronic stopping power and the energy loss straggling for low velocity $\mathrm{H}-, \mathrm{He}-, \mathrm{Li}$ - and Be-like projectiles in the degenerate electron gas are reported. The Hartree-Fock-Slater description of the projectile and the dielectric function method were used. The size parameter $Z_{\min }$ of the charge distributions calculated from a variational principle depends on the characteristics of the medium. The stopping and straggling effective charges $Z_{\text {ef }}$ of a projectile were analysed. They were found to differ with each other and to depend on the one-electron radius $r_{\mathrm{s}}$, on the projectile atomic number $Z_{i}$ and on the number of electrons $N_{i}$ carried by the projectile.
\end{abstract}

PACS numbers: 71.45.Gm, 34.50.Bw

\section{Introduction}

After crossing the surface of a solid a slow atomic nucleus captures electrons forming intermediate electronic configurations up to being almost completely neutralised as it stops. This configuration strongly modifies the electronic stopping power and the energy loss straggling for the ion beam, which are important in analysis of distribution and lattice localisation of implanted atoms or in analysis of surface structure. The most important works in this field [1-5] were related to analysis of the stopping and straggling of an atomic nucleus as a projectile and neglecting thus an effect of its electronic configuration. A common feature of these theories is proportionality of the stopping power to velocity $v$ and the energy loss straggling to $v^{2}$. The target and the projectile dependencies contained in the proportionality factor are model-dependent and they are different. The target, which consists of the free electron gas (at $T=0 \mathrm{~K}$ ), is characterised by the one-electron radius $r_{\mathrm{s}}\left(n=3 / 4 \pi r_{\mathrm{s}}^{3} a_{0}^{3}\right.$ is the electron gas density). The projectile consists of an

*Supported by Uniwersytet Eódzki, grant 505/576 (1998).

†e-mail: moneta@krysia.uni.lodz.pl 
atomic nucleus of atomic number $Z_{i}$, moving slowly with velocity $v$ and carrying $N_{i}$ electrons.

In this paper $m, e, a_{0}$, and $v_{0}$ are the electron rest mass, the elementary charge, the Bohr radius, and the Bohr velocity, respectively. Atomic units are used throughout.

\section{Calculation procedure}

The probability for transfer of the energy $\omega$ and the momentum $k$ to a degenerate free electron gas from a projectile is described within the random phase approximation (RPA) by the equilibrium dielectric function $\epsilon(k, \omega)$. Commonly the dimensionless parameters $z=k / 2 k_{\mathrm{F}}, u=\omega / k v_{\mathrm{F}}$ and $\chi^{2}=r_{\mathrm{s}} / \pi \alpha$ are used, where $k \dot{\mathrm{F}}=\alpha / a_{0} r_{\mathrm{s}}$ is the modulus of the Fermi wave vector and $\alpha=(9 \pi / 4)^{1 / 3}$. The electronic stopping cross-section $S$ and the straggling parameter $\Omega^{2}$ (per free electron) for a projectile of velocity $v$ are given by [1,2]:

$$
S=\frac{1}{n} \frac{\mathrm{d} E}{\mathrm{~d} x}=\frac{4 \pi e^{4}}{m v^{2}} Z_{i}^{2} L_{1}, \quad \frac{1}{n} \frac{\Omega^{2}}{x}=\frac{4 \pi e^{4}}{m v^{2}} Z_{i}^{2} L_{2},
$$

where

$$
L_{m}=\left(4 E_{\mathrm{F}}\right)^{m-1} \frac{6}{\pi \chi^{2}} \int_{0}^{v / v_{\mathrm{F}}} \mathrm{d} u u^{m} \int_{0}^{\infty} \mathrm{d} z z^{m} Z^{2}(z) \Im\left[\frac{1}{\epsilon(u, z)}-1\right],
$$

where the dielectric function reads $\epsilon(u, z)=1+\left(\chi^{2} / z^{2}\right)\left[f_{1}(u, z)+\mathrm{i} f_{2}(u, z)\right], E_{\mathrm{F}}=$ $\alpha^{2} / 2 r_{\mathrm{s}}^{2}$ is the Fermi energy, and the factor $4 \pi e^{4} / m v^{2}=4 \pi e^{2} a_{0}\left(v_{0} / v\right)^{2}$. The form factor $Z^{2}(z)$ is the Fourier transform of the spatial electron distribution on the projectile [6-8] being a sum of the screening component $Z_{\mathrm{s}}^{2}$ and the anti-screening component $Z_{\mathrm{a}}^{2}$,

$$
Z^{2}(z)=Z_{\mathrm{s}}^{2}(z)+Z_{\mathrm{a}}^{2}(z)=\left[1-\rho(z) N_{i} / Z_{i}\right]^{2}+\left\{1-[\rho(z)]^{2}\right\} N_{i} / Z_{i}^{2}
$$

where $\rho(z)$ is the one-electron form factor.

Explicitly Eq. (2) takes the form

$$
L_{m}=-\left(4 E_{\mathrm{F}}\right)^{m-1} \frac{6}{\pi} \int_{0}^{v / v_{\mathrm{F}}} \mathrm{d} u u^{m} \int_{0}^{\infty} \mathrm{d} z \frac{z^{m+2} Z^{2}(z) f_{2}(u, z)}{\left[z^{2}+\chi^{2} f_{1}(u, z)\right]^{2}+\left[\chi^{2} f_{2}(u, z)\right]^{2}} .
$$

The coilduction electrons of a solid screen the quasi-static electric potential of a slow projectile due to dielectric response. Provided the speed of the atom is lower than the Fermi velocity $v_{\mathrm{F}}$, this screening can be approximately described in terms of the Coulomb potential between charges with the screening function $\exp \left(-r k_{\mathrm{TF}}\right)$, where the Thomas-Fermi wave number $k_{\mathrm{TF}}$ is related to the Fermi wave number as $k_{T F}^{2}=4 k_{F} / \pi a_{0}$. In this approximation we neglected to account for the full Lindhard dielectric function. More precisely, instead of the exponential decay the screening function displays rather the Freedel oscillations $V \propto \cos \left(r k_{T F}\right) / r^{3}$ caused by sharpness of the Fermi surface at $T=0 \mathrm{~K}$.

The size parameter of bound electrons cloud can be determined either statistically or quantum mechanically. We want to determine stopping and straggling characteristics of the electron gas for an extended charge projectile by means of a size parameter of the charge distribution. This parameter is modified when the projectile enters a solid. When we deal with slow heavy projectiles carrying 
many electrons, then the statistical description and the density functional method are justified. When we consider projectiles with a small number of electrons the Hartree-Fock-Slater (HFS) description must be used. The intermediate region cannot be treated analytically.

We determine the volume parameter $\lambda$ from the condition of minimum for the expectation value $\bar{H}$ of the total self-consistent, Hamiltonian given (in hartree units) by

$$
H=-\frac{1}{2} \sum_{j} \Delta_{j}-Z_{i} \sum_{j} \frac{\exp \left(-r_{j} k_{\mathrm{TF}}\right)}{r_{j}}+\frac{1}{2} \sum_{j \neq k} \frac{\exp \left(-k_{\mathrm{TF}}\left|r_{j}-r_{k}\right|\right)}{\left|r_{j}-r_{k}\right|}
$$

with the orthonormal, one-electron trial eigenfunctions forming the HFS determinant ( $\lambda=a_{0} / Z$ or $Z$ alone are variational parameters)

$$
\begin{aligned}
& u_{1 s}(r)=\pi^{-1 / 2}(\lambda)^{-3 / 2} \exp (-r / \lambda) \\
& u_{2 s}(r)=\pi^{-1 / 2}(2 \lambda)^{-3 / 2}(1-r / 2 \lambda) \exp (-r / 2 \lambda) .
\end{aligned}
$$

The expectation values for the total Hamiltonian describing different $1 s 2 s$ configurations can be calculated as

$$
\begin{aligned}
& \bar{H}\left(\mathrm{H} \equiv 1 s^{1}\right)=E_{1 s}, \\
& \bar{H}\left(\mathrm{He} \equiv 1 s^{2}\right)=2 E_{1 s}+V_{1 s 1 s} \\
& \bar{H}\left(\mathrm{Li} \equiv 1 s^{2} 2 s^{1}\right)=2 E_{1 s}+E_{2 s}+V_{1 s 1 s}+2 V_{1 s 2 s}-A_{1 s 2 s} \\
& \bar{H}\left(\mathrm{Be} \equiv 1 s^{2} 2 s^{2}\right)=2 E_{1 s}+2 E_{2 s}+V_{1 s 1 s}+V_{2 s 2 s}+4 V_{1 s 2 s}-2 A_{1 s 2 s}
\end{aligned}
$$

where the eigenenergies $(E)$, the Coulomb $(V)$, and the exchange $(A)$ integrals are given in Appendix.

The Fourier transform of the spatial electron distribution on the projectile carrying $N_{1 s}$ electrons in the $1 s$ state and $N_{2 s}$ electrons in the $2 s$ state $\left(N_{i}=\right.$ $N_{1 s}+N_{2 s}$ ) from Eq. (6) reads

$$
\begin{aligned}
& N_{i} \rho(z)=N_{1 s} \rho_{1 s}(2 \phi)+N_{2 s} \rho_{2 s}(2 \phi) \\
& =N_{1 s} \frac{1}{\left(1+\phi^{2}\right)^{2}}+N_{2 s} \frac{1-3(2 \phi)^{2}+2(2 \phi)^{4}}{\left[1+(2 \phi)^{2}\right]^{2}}
\end{aligned}
$$

where $\phi=k_{\mathrm{F}} \lambda z$.

\section{Results and discussion}

We carried out calculations for extended charge projectiles moving slowly in the uniform electron gas. We get analytical results for the stopping power and for the energy loss straggling for the gas described by Lindhard's dielectric function $\epsilon(u, z)=1+\left(\chi^{2} / z^{2}\right)\left[f_{1}(u, z)+\mathrm{i} f_{2}(u, z)\right][1,2]$. The $L_{m}$ functions of Eq. (4) are expressed as

$$
L_{m}=\left(3 E_{\mathrm{F}}\right)^{m-1}\left(\frac{v}{v_{\mathrm{F}}}\right)^{m+2} C_{m}, \quad C_{m}=\int_{0}^{1} \mathrm{~d} z z^{m+2} \frac{Z^{2}(z)}{\left[z^{2}+\chi^{2} f_{1}(0, z)\right]^{2}} .
$$


The variable $u=m \omega / k k_{\mathrm{F}}=\bar{h} \omega / z E_{\mathrm{F}}$ measures the energy $\bar{h} \omega$ (in units of the Fermi energy $E_{\mathrm{F}}$ ) transferred from the projectile to the medium. For slow projectiles the energy transfer is very low, i.e. $u \ll 1$, therefore the approximation leading from Eq. (4) to Eq. (9) is justified. In this case the approximation $f_{1}\left(0, z^{*}\right)=1-z^{2} / 3$ can be used, therefore the denominator in Eq. (9) reads $\left[z^{2}+\right.$ $\left.\chi^{2}\left(1-z^{2} / 3\right)\right]^{2}=\left(\chi^{2} / \chi^{\prime 2}\right)^{2}\left(z^{2}+\chi^{\prime 2}\right)^{2}$, where $\chi^{\prime 2}=\chi^{2} /\left(1-\chi^{2} / 3\right)$. For real metals $1.5<r_{\mathrm{s}}<5.8$, therefore $0.50<\chi<0.98$ and $0.52<\chi^{\prime}<1.19$.

From Eq. (1), in atomic units (hartree/ $a_{0}$ and hartree ${ }^{2} / a_{0}$ ), we get

$$
\frac{\mathrm{d} E}{\mathrm{~d} x}=\frac{4}{3 \pi} \frac{v}{v_{0}} Z_{i}^{2} C_{1}, \quad \frac{\Omega^{2}}{x}=\frac{9}{r_{\mathrm{s}}^{3}}\left(\frac{v}{v_{0}}\right)^{2} Z_{i}^{2} C_{2} .
$$

For heavier ions carrying many electrons we get analytical formulas for $C$ 's cited in the previous paper [9].

In the case of projectiles carrying a small number of electrons in the $1 s$ and $2 s$ states and described by the form factor from $\mathrm{Eq}$. (3) we get the lunctions $C_{1}$ and $C_{2}$ in closed analytical forms. They depend on $\left(Z_{i}, r_{s}, N_{1 s}, N_{2 s}\right)$ parameters. Due to their complicated forms they will not be presented here, but can be obtained from the author on request. The analytical result allows for power expansion, contrary to the direct numerical integration in Eq. (9). $L_{1}$ and $C_{m}$ are dimensionless and $L_{2}$ is expressed in atomic hartree units.

This formulas are directly reduced to the case of an atomic nucleus by setting $N_{i}=0$. From Eq. (9) we get

$$
\begin{aligned}
& C_{1}=0.5\left[-\frac{1}{1+\chi^{\prime 2}}+\ln \left(1+\frac{1}{\chi^{\prime 2}}\right)\right], \\
& C_{2}=1+0.5\left(\frac{\chi^{\prime 2}}{1+\chi^{\prime 2}}-3 \chi^{\prime} \arctan \frac{1}{\chi^{\prime}}\right) .
\end{aligned}
$$

When a projectile with few electrons moves in the vacuum then $\gamma=0$, and then the $\bar{H}$ 's of Eq. (7) reach minima (calculated from the requirement $\partial \bar{H} / \partial Z=0$ ) at $Z_{\min }$ equal to $Z_{\mathrm{H}}=Z_{i}, Z_{\mathrm{He}}=Z_{i}-0.3125, Z_{\mathrm{Li}}=Z_{i}-0.45458$ and $Z_{\mathrm{Be}}=Z_{i}-0.6284$, for hydrogen-, helium-, lithium- and berilium-like electronic configurations, respectively. The corresponding energy minima are: $\bar{H}_{\mathrm{He}}=-Z_{\mathrm{He}}^{2}$, $\bar{H}_{\mathrm{Li}}=-(9 / 8) Z_{\mathrm{Li}}^{2}, \bar{H}_{\mathrm{Be}}=-(5 / 4) Z_{\mathrm{Be}}^{2}$ in hartree units. When the projectile moves slowly in a solid, keeping all the time a stable electronic configuration, these parameters are modified due to interaction with electron gas. For each electronic configuration the screening parameter $\lambda\left(Z_{\min }\right)$ was calculated by taking numerically minimum of the appropriate $\bar{H}$ from Eq. (7), therefore $\lambda$ depends on $Z_{i}, N_{i}$, and additionally on $r_{\mathbf{s}}$. Subsequently $\lambda$ was used in Eqs. $(8,3)$.

This solid state effect on screening was shown in Fig. 1. We plot the difference $Z_{i}-Z_{\min }$ for Be-like projectiles; as a function of $Z_{i}$ and $r_{\mathrm{s}}$. It is obvious that all such $Z_{\min }$ functions tend to the above limits in the dilute electron gas, as $r_{\mathrm{s}}$ is large. For a dense electron gas (small $r_{\mathrm{s}}$ ) the functions are larger than the limits, which means stronger screening of the projectile nucleus interaction by the medium. This screening is more important at low $Z_{i}$. 


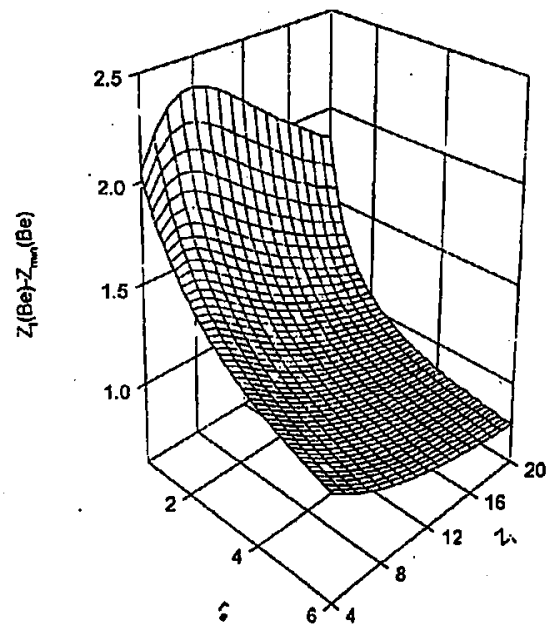

Fig. 1. The parameter $Z_{\min }$ calculated for Be-like projectiles of atomic number $Z_{i}$. The atomic and the screening effects are included.

The common feature of the present and other results is that the stopping power $S$ is proportional to $v$ and the energy loss straggling $\Omega^{2}$ to $v^{2}$ at low ion velocity $v$. The differences are model-dependent and are related to the coefficient of proportionality $C\left(Z_{i}, r_{\mathrm{s}}, N_{1 s}, N_{2 s}\right)$ which incorporates both the target parameter $r_{\mathrm{s}}$ and the projectile parameters $Z_{i}, N_{1 s}$, and $N_{2 s}$. They cannot be further simplified or separated even after power expansion.

In order to perform calculations we assume a stable in time, frozen charge distribution on the projectile. Within this model $[\kappa, 7,9]$ the projectile charge in the Fourier space which contributes to Eq. (9) is .. sum of the screening component $\left[1-\rho(z) N_{i} / Z_{i}\right]^{2}$ and the anti-screening component $\left\{1-[\rho(z)]^{2}\right\} N_{i} / Z_{i}^{2}$. As long as we deal with slow, heavy projectiles, considering only the screening component is justified. For light and neutral projectiles the anti-screening must be taken into account, since it enhances the stopping and straggling by about $10 \%$. From Eq. (3) we find that in the low momentum transfer limit $Z^{2}(0)=\left[1-N_{i} / Z_{i}\right]^{2}$ we get reduction of soft, distant collisions contribution to the loss process. The large momentum transfer limit $Z^{2}(\infty)=1+N_{i} / Z_{i}^{2}$ gives enhancement of the contribution from hard, close collisions due to excitations of the electron gas by projectile electrons.

In order to analyse results we should realise that dependence of $C$ 's on $Z_{i}$ means that the proportionality of the energy loss and the straggling to $Z_{i}^{2}$, correct for a point charge, is broken in a case of an extended charge, and the projectile excites the medium as a stable charge configuration. The behaviour of $C$ 's with $r_{\mathrm{s}}$ can be understood by noting that the energy absorbed by the electron gas on collective excitations drops as $r_{\mathrm{s}}^{-3 / 2}$ and the number of electrons subjected to the single particles excitations are related to the density of states below the Fermi level $E_{\mathrm{F}}$. 
From the previous paper [10] (when statistical description of the projectile electrons is applied), if we expand $C_{1}$ and $C_{2}$ in a power series we find that for a dense medium and for a heavy projectile $C_{1} \propto r_{\mathrm{s}}^{4} Z_{i}^{-4 / 3}$ and $C_{2} \propto r_{\mathrm{s}}^{2} Z_{i}^{-2 / 3}$. From Eq. (10) we get $\mathrm{d} E / \mathrm{d} x \propto Z_{i}^{2 / 3}$ and $\Omega^{2} / x \propto Z_{i}^{4 / 3}$, respectively.

For the energy loss analysis the concept of effectivc charge is applied $[6,7,9]$. It relates the stopping and straggling produced by a given projectile to the same characteristics produced by the projectile atomic nucleus. We define the effective charge for the stopping $Z_{\text {ef1 }}$ and for straggling $Z_{\text {ef2 }}$ separately as

$$
Z_{\text {ef } m}=\sqrt{C_{m}\left(Z_{i}, r_{\mathrm{s}}, N_{1 s}, N_{2 s}\right) / C_{m}\left(Z_{i}, r_{\mathrm{s}}, 0, \mathcal{\cup}\right)}
$$

For a point charge $Z_{\text {ef } m}=1$. An independence of $Z_{\text {ef } m}$ on $Z_{i}$ means that the Bethe $Z_{i}^{2}$ scaling is only accidentally valid for both stopping and straggling. This scaling is related to the same contribution of close and distant collisions in the process of energy transfer to the electron gas. In the static case the result $Z_{\text {ef }}<1$ means that projectile electrons screen the Coulomb potential of the projectile nucleus. As an example we have shown in Fig. 2 the stopping power effective charge $Z_{\text {ef } 1}$ for Li-like projectiles. In the dilute electron gas $\left(r_{\mathrm{s}}=6\right)$ and for low projectile atomic number $Z_{i}$ the effective charge is much smaller than unity, $Z_{\text {ef } 1} \ll 1$. For large $Z_{i}$ it tends slowly to unity.

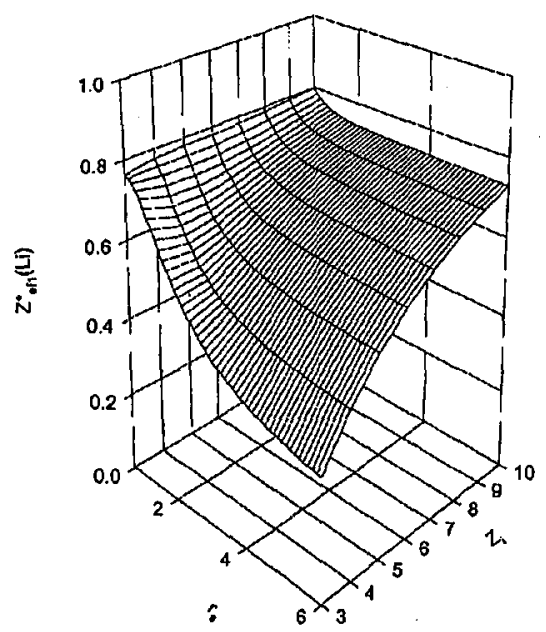

Fig. 2. The stopping effective charge for Li-like projectiles.

It is of interest to see how large are the atomic and the electron gas screening contributions calculated for $Z_{\min }$, when compared to the $Z_{\min }=Z_{i}$ case. The difference between the stopping power effective charges $Z_{\text {ef1 }}$ are plotted in Fig. 3 as a function of $r_{\mathrm{s}}$ and $Z_{i}$ for Be-like projectiles. It reaches maximum at $r_{\mathrm{s}}=0.7$ for small $Z_{i}$ and rapidly decreases to zero as $Z_{i}$ increases. The energy straggling effective charge $Z_{\text {ef } 2}$ displays the similar behaviour. 


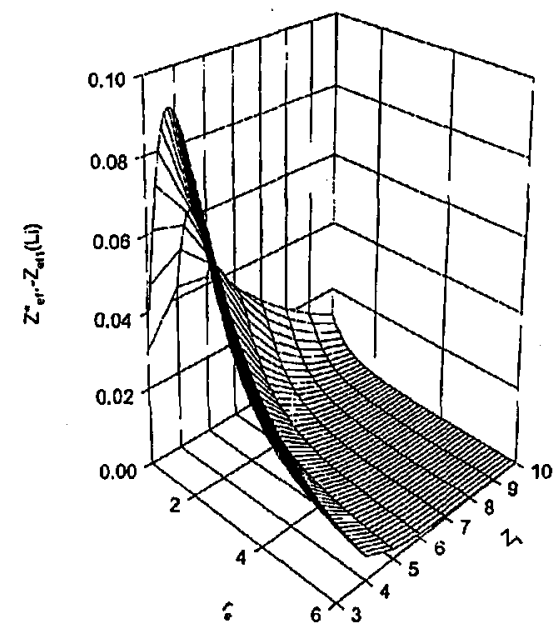

Fig. 3. The effect of switching on the atomic and the screening contributions to the calculation of the stopping effective charge for Li-like projectiles. $Z_{\text {ef } 1}$ is calculated by taking $Z_{\min }=Z_{i}$.

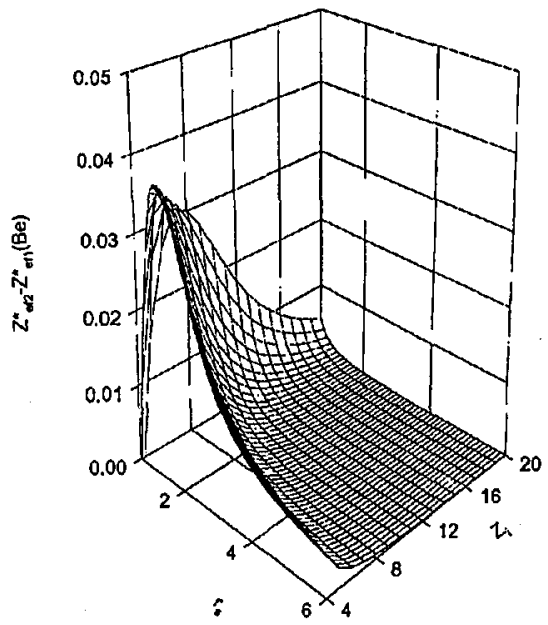

Fig. 4. Difference between the straggling effective charge $Z_{\mathrm{ef} 2}$ and the stopping effective charge $Z_{\text {ef1 }}$ for Be-like projectiles.

In Fig. 4 the differences between the effective charges for straggling and for stopping $Z_{\mathrm{ef} 2}-Z_{\mathrm{ef} 1}$ is shown for Be-like projectiles as a function of $r_{\mathrm{s}}$ versus $Z_{i}$. The atomic and the solid state effects were included here through $Z_{\min }$ calculated by taking minimum of $\bar{H}\left(\mathrm{Be}=1 s^{2} 2 s^{2}\right)$ in Eq. (7). The differences between both effective charges are $r_{\mathrm{s}}$ dependent (they amount to $3.5 \%$ ) for small $Z_{i}$ and tend to zero as $Z_{i}$ increases. This interesting feature is caused by a structure of the integrals in Eq. (9). 


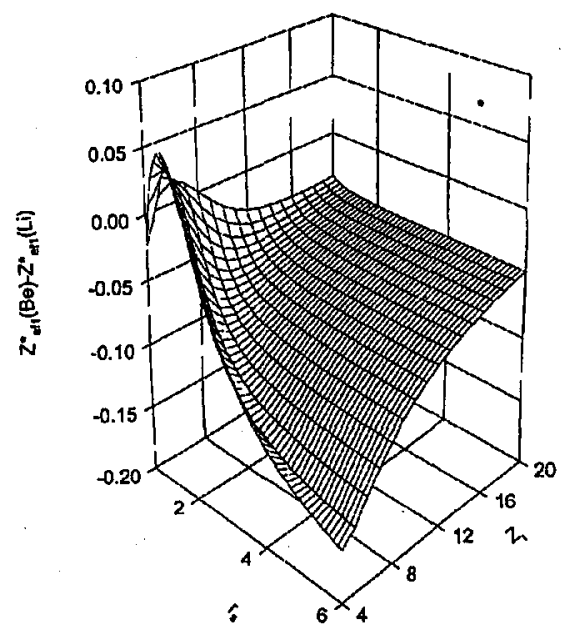

Fig. 5. The influence of one additional $2 s$ electron on the stopping power effective charge between Li-like and Be-like projectiles.

Another interesting question is how the effective charge will change, when we add (or remove) one electron to (from) the projectile. In Fig. 5 we have shown the difference between the stopping effective charge $Z_{\mathrm{ef} 1}$ for a Be-like projectile of the net charge $Z_{i}-4$ and the stopping effective charge $Z_{\text {ef } 1}$ for a Li-like projectile of the net charge $Z_{i}-3$. Nearly for all $r_{\mathrm{s}}$ and $Z_{i}, Z_{\text {ef } 1}(\mathrm{Be})<Z_{\text {ef } 1}(\mathrm{Li})$. Asymptotically, for large $Z_{i}$, adding one electron to the projectile causes negligible decrease in the effective charge. For a dilute electron gas and for small $Z_{i}$ this difference reaches 0.2 . For a dense $\left(r_{\mathrm{s}}=0.7\right)$ electron gas the situation is opposite: $Z_{\text {ef } 1}(\mathrm{Be})>$ $Z_{\text {ef1 }}(\mathrm{Li})$ which means that a Be-like projectile transfers the energy to the medium more effectively than a Li-like projectile.

\section{Conclusions}

Result of the calculation for the electronic stopping power and the energy loss straggling of free electron gas for low velocity $\mathrm{H}-$, $\mathrm{He}-, \mathrm{Li}$ - and $\mathrm{Be}-$ like projectile was presented. The Hartree-Fock-Slater description of the projectile in a solid was used. The size parameter $\lambda$ (or $Z$ ) was determined from a variational principle and shown to depend on $r_{\mathrm{s}}, \mathrm{Z}_{\mathrm{i}}, \mathrm{N}_{1 \mathrm{~s}}$, and $N_{2 s}$. The anti-screening correction was included and found to be important for small $Z_{i}$. The dependence of the effective ion charges on the target electron gas density $r_{s}$ and on the projectile atomic number $Z_{i}$ and the number of electrons on the projectile was discussed.

\section{Appendix}

The eigenenergies $(E)$, the Coulomb $(V)$, and the exchange $(A)$ integrals were calculated as $\left(\gamma=k_{\mathrm{TF}} \lambda\right.$ and $\left.\gamma^{\prime}=2 \gamma / 3\right)$

$$
E_{1 s}=\frac{e^{2}}{a_{0}}\left[\frac{Z^{2}}{2}-\frac{Z_{i} Z}{(1+\gamma / 2)^{2}}\right],
$$




$$
\begin{aligned}
& E_{2 s}=\frac{e^{2}}{a_{0}}\left[\frac{Z^{2}}{8}-\frac{Z_{i} Z}{4(1+\gamma)^{2}}\left(2-\frac{4}{(1+\gamma)}+\frac{3}{(1+\gamma)^{4}}\right)\right] \\
& V_{1 s 1 s}=\frac{e^{2}}{a_{0}} Z \frac{5+\gamma+\gamma^{2} / 4}{8(1+\gamma / 2)^{4}} \\
& V_{2 s 2 s}=\frac{e^{2}}{a_{0}} Z \frac{77+104 \gamma+227 \gamma^{2}+144 \gamma^{3}+123 \gamma^{4}+40 \gamma^{5}+5 \gamma^{6}}{512(1+\gamma)^{4}}, \\
& V_{1 s 2 s}=\frac{e^{2}}{a_{0}} Z \frac{4}{81} \frac{17+4 \gamma+21 \gamma^{2}+8 \gamma^{3}+\gamma^{4}}{(1+\gamma)^{4}(2+\gamma)^{2}} \\
& A_{1 s 2 s}=\frac{e^{2}}{a_{0}} Z \frac{16}{729} \frac{3-25 \gamma^{\prime 2}+150 \gamma^{4}-256 \gamma^{\prime 5}+150 \gamma^{\prime 6}-25 \gamma^{\prime 8}+3 \gamma^{\prime 10}}{3\left(1-\gamma^{\prime 2}\right)^{6}} .
\end{aligned}
$$

\section{References}

[1] J. Lindhard, K. Dam. Vid. Selsk. Mat. Fys. Med. 28 (1954).

[2] J. Lindhard, A. Winter, K. Dam. Vid. Selsk. Mat. Fys. Med. 34 (1964).

[3] J. Lindhard, M. Scharf, Phys. Rev. A 4, 562 (1971); K. Dam. Vid. Selsk. Mat. Fys. Med. 27 (1953).

[4] O.B. Firsov, Zh. Eksp. Teor. Fiz. 36, 1517 (1959).

[5] P. Hvelplund, K. Dam. Vid. Selsk. Mat. Fys. Med. 38 (1971).

[6] M. Moneta, Nucl. Instrum. Methods Phys. Res. B 115, 340 (1996).

[7] M. Moneta, Acta Phys. Pol. B 24, 2033 (1993).

[8] M. Moneta, Acta Phys. Pol. A 89, 581 (1996).

[9] M. Moneta, Acta Phys. Pol. A 92, 1169 (1997). 